\title{
Polyembryoma of the testis: a report of two cases dominant within mixed germ cell tumors and review of gonadal polyembryomas
}

\author{
Jennifer N Stall ${ }^{1}$ and Robert H Young ${ }^{2}$ \\ ${ }^{1}$ Hospital Pathology Associates, Virginia Piper Cancer Institute, Abbott Northwestern Hospital, Minneapolis, \\ MN, USA and ${ }^{2} J a m e s$ Homer Wright Pathology Laboratories, Massachusetts General Hospital, Harvard \\ Medical School, Boston, MA, USA
}

\begin{abstract}
Two testicular mixed germ cell tumors, from men of 21 and 41 years, in which polyembryoma predominated are described. A literature review uncovered an additional five testicular and nine ovarian cases. One tumor occurred in a 60-year-old man, but all others occurred within the typical age range of gonadal germ cell tumors. One male presented with gynecomastia and one female with sexual precocity, but all otherwise had standard clinical manifestations. These tumors are typically large with non-specific gross features, but a few have a prominent hemorrhagic appearance. No tumor is known to have been entirely composed of embryoid bodies, the unit upon which the diagnosis of polyembryoma is based. The most common additional germ cell tumor component is teratoma, present in the great majority of cases. An approximately equal smaller number of tumors are associated with embryonal carcinoma and yolk sac tumor, which arise as overgrowths of embryonal carcinoma and yolk sac-type epithelium from within the parent embryoid body. Rarely there is choriocarcinoma, and syncytiotrophoblast and hepatoid cells are occasionally present. The microscopic features of the tumors vary according to the arrangement of embryoid bodies with other elements, the prominence of associated typically myxoid to edematous stroma, and the degree to which embryoid bodies are perfectly or imperfectly formed. Although its presence in a gonadal mixed germ cell tumor is probably not associated with any special behavior, its unique features should result in polyembryoma being recorded, particularly when present in significant amount. Furthermore, awareness of its features may facilitate recognition, particularly when seen at metastatic sites or extra-gonadal sites of primary germ cell neoplasia. Whether polyembroma should be considered a distinctive pattern of mixed germ cell neoplasia or a particular variant of high-grade immature teratoma is considered, herein, and arguments can be made in favor of each viewpoint.

Modern Pathology (2017) 30, 908-918; doi:10.1038/modpathol.2017.25; published online 21 April 2017
\end{abstract}

The spectrum of microscopic appearances in both teratomatous and nonteratomatous germ cell tumors is remarkably diverse. Perhaps the most striking finding is that of embryoid bodies. First drawn to attention by the French investigator Peyron ${ }^{1}$ in the 1930s, these unique neoplastic formations recapitulate embryonic life circa 13-18 days of gestation. Proliferations of these structures have been designated for some decades as 'polyembryoma.' In a recent review, ${ }^{1}$ we considered the history of this neoplasm, the timeline of contributions on it, its morphologic features, and Peyron's life. Although

Correspondence: Dr JN Stall, MD, Hospital Pathology Associates, 2800 10th Ave S Suite 2200, Minneapolis, MN 55407, USA.

E-mail: jennifer.stall@allina.com

Received 8 February 2017; accepted 9 February 2017; published online 21 April 2017 individual examples from the literature were cited, they were not critically analyzed, nor were outcome and potential differences in the sexes considered. When preparing that contribution, we had access to the slides of one unpublished testicular example and have since reviewed slides of another. Although one study found embryoid bodies in $\sim 40 \%$ of testicular germ cell tumors, ${ }^{2}$ neoplasms in which they predominate, and hence have a meaningful polyembryoma component are rare. This, and the still relatively limited documentation of the fascinating morphologic aspects of this tumor, prompted us to fully analyze the microscopic features of the two testicular cases available to us and conduct a detailed review of previously reported cases in both gonads. In addition, we discuss the differing views concerning categorization of this tumor, represented by the divergent opinions expressed in the recent 
World Health Organization classifications of gonadal tumors. In the work considering the testis the neoplasm was considered a form of mixed germ cell tumor; ${ }^{3}$ in that considering the ovary it was considered a pattern of high-grade immature teratoma. ${ }^{4}$

\section{Materials and methods}

Case 1 was a routinely encountered case, in which 30 hematoxylin and eosin stained slides of the tumor were available for review. Case 2 was seen in consultation by Dr Robert E. Scully many years ago. At the time of our recent essay ${ }^{1}$ the slides were missing, but 29 hematoxylin and eosin stained slides were subsequently made available to us (see Acknowledgements). We have also had the opportunity to review two previously reported ovarian polyembryomas, both present in the files of the Robert E. Scully Collection at the Massachusetts General Hospital. The specific cases are noted in the discussion.

\section{Case reports}

\section{Case 1}

A 41-year-old previously healthy Caucasian male presented with swelling of the right testis. Physical examination revealed a firm nontender mass, and laboratory evaluation demonstrated an elevated serum AFP at $746 \mathrm{ng} / \mathrm{ml}$ (reference range $<8.5 \mathrm{ng}$ / $\mathrm{ml}$ ) and beta-human chorionic gonadotropin (BHCG) at $43.2 \mathrm{IU} / \mathrm{ml}$ (reference range $<5 \mathrm{IU} / \mathrm{ml}$ ). The patient underwent a right orchiectomy. The testicular parenchyma was largely replaced by a $6.0 \times 5.0 \times 1.8 \mathrm{~cm}$ well-circumscribed red to brown nodular mass with a few fibrous septa and focal cystic change (Figure 1).

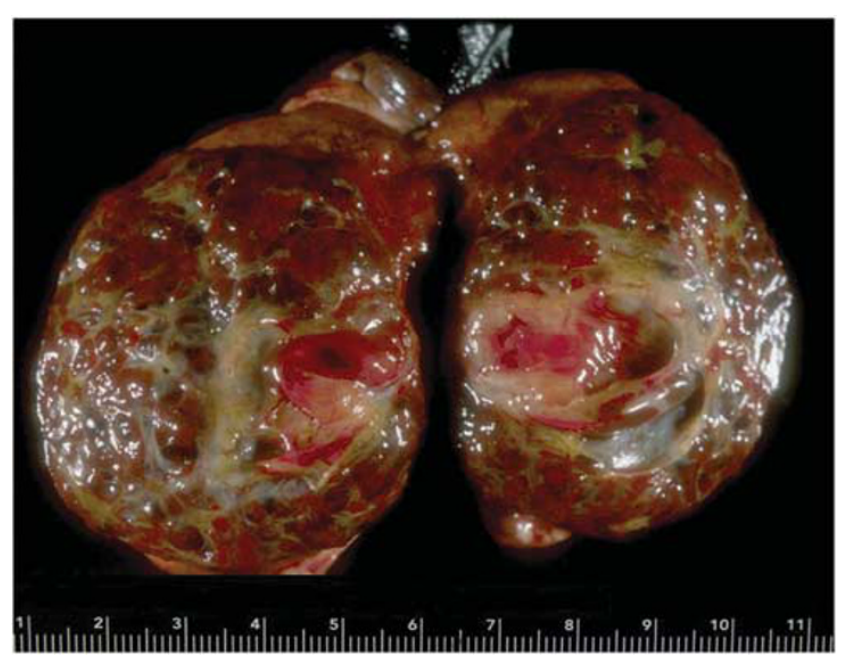

Figure 1 Gross of Case 1. The testicular parenchyma was largely replaced by a hemorrhagic multinodular solid mass, which had a focally conspicuous lobulated sectioned surface. Interspersed fibrous bands and a few cysts (right) were present.
Histologic examination demonstrated a predominant polyembryoma component, which accounted for $\sim 90 \%$ of the tumor (Figure 2). Numerous embryoid bodies were present on a background of a prominent edematous to myxoid stroma. The stromal component accounted for the majority of the lesional tissue, which also contained scattered foci of hemorrhage that were often conspicuous (Figure 2a and b). Focally, the embryoid bodies and associated stroma formed discrete lobules, but for the most part the lobules were ill-defined. The structure of the embryoid bodies varied in appearance. Some were relatively well-formed with an easily recognizable focus of embryonal carcinoma-type epithelium forming the so-called germ disc underlain by thinner more delicate appearing epithelium of yolk-sac type (Figure 2d). However, the majority of the embryoid bodies were poorly formed with a more haphazard arrangement of the embryonal and yolk-sac epithelial components; the two components still grew in combination, enabling them to be recognized as embryoid bodies. An additional focally striking component of the tumor was cysts lined by flattened epithelium that appeared to represent markedly dilated yolk-sac vesicles as minor degrees of dilation of the vesicle in clearly evident embryoid bodies was frequent. Rare syncytiotrophoblast giant cells were present in association with some embryoid bodies.

In many of the embryoid bodies, the yolk-sac epithelium proliferated to form isolated foci that on high power would be consistent with yolk-sac tumor. However, for the most part these foci did not expand beyond the confines of the embryoid body and thus, by convention, did not warrant a diagnosis of yolk-sac tumor. ${ }^{1}$ In $\sim 5 \%$ of the tumor, aggregates of yolk-sac epithelium expanded beyond the embryoid body in aggregates of $3 \mathrm{~mm}$ or more and warranted a secondary diagnosis of a minor yolk-sac tumor component. The remaining $5 \%$ of the tumor was composed of mature teratomatous elements without immature foci. Background germ cell neoplasia in situ was not appreciated in the adjacent uninvolved testis, which was histologically unremarkable. The tumor was confined to the testis without evidence of lymphovascular invasion.

Within one week of surgery, the serum AFP dropped to $236 \mathrm{ng} / \mathrm{ml}$ and BHCG to $<2.0 \mathrm{IU} / \mathrm{ml}$. The serum AFP continued to decline, reaching a normal level within seven weeks. After six months, the serum AFP became elevated and chest imaging demonstrated a new $1.4 \mathrm{~cm}$ lesion in the left lower lobe. The patient underwent chemotherapy with a decrease in size of the lesion to $1.0 \mathrm{~cm}$, as well as decreased serum AFP. A left lower lobe wedge resection was performed, revealing a $1.5 \times 1.1 \times$ $1.0 \mathrm{~cm}$ well-circumscribed white firm multicystic mass focally abutting the pleura. The entire tumor was submitted for histologic examination, which demonstrated predominantly fibrosis with rare mature epithelial elements, consistent with treated metastatic teratoma. Immature elements, 

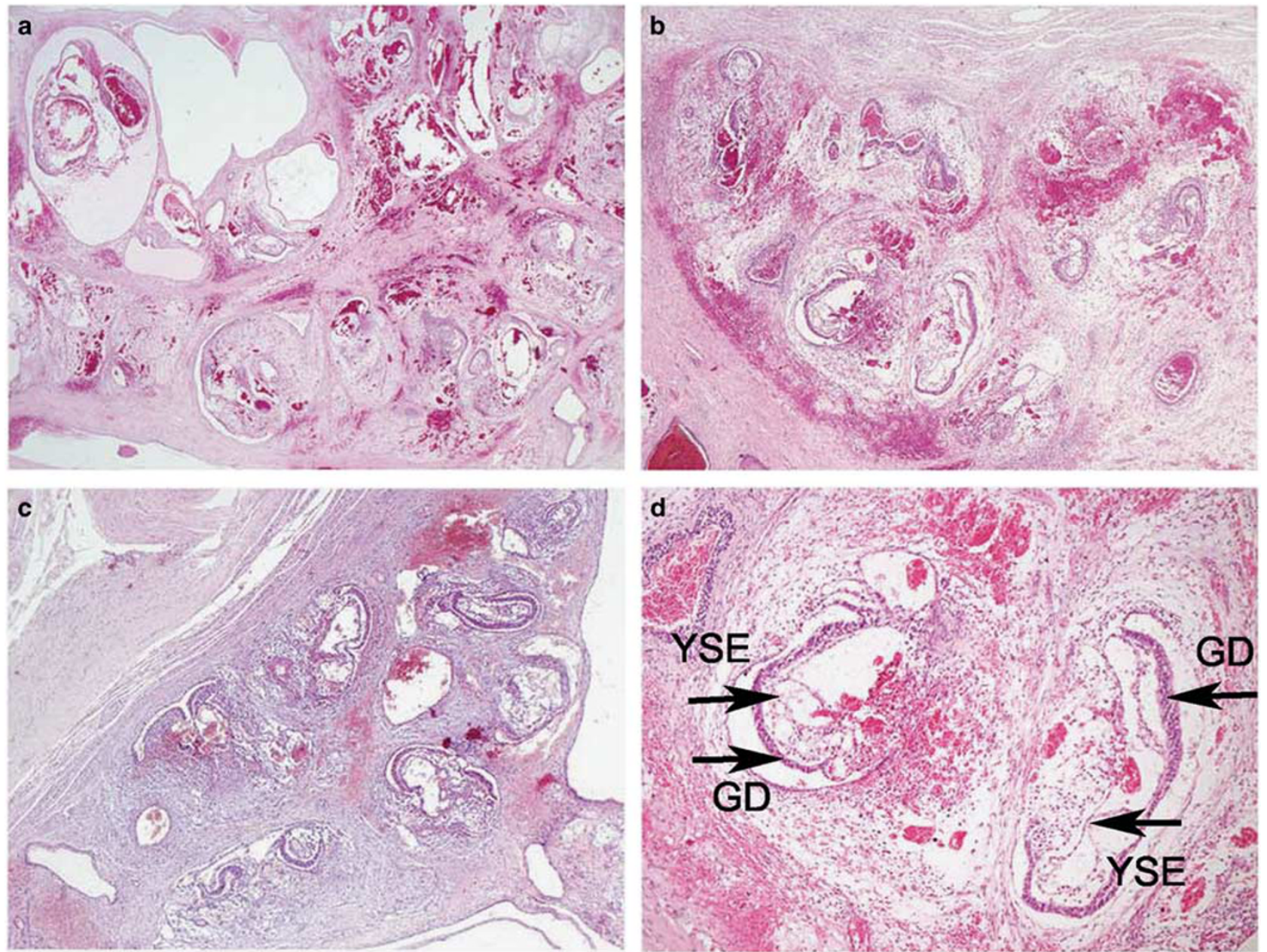

Figure 2 Representative low to medium power views of hematoxylin and eosin stained sections of a testicular polyembryoma (Case 1). All images contain darkly staining germ discs composed of embryonal carcinoma-type epithelium with underlying thin wispy yolk-sac epithelium (best seen in d) with the latter image demonstrating two well-formed embryoid bodies. Prominent fibrous (a, b) to myxoid (c) stroma surrounds the clusters of embryoid bodies. Scattered foci of hemorrhage (a-d) and cysts (a, upper left) may be striking in some polyembryomas. Arrows point to the germ disc (GD) and yolk-sac epithelium (YSE) in d.

including embryoid bodies, were not identified. The patient subsequently remained well for the following four years, at that time he was lost to follow-up.

\section{Case 2}

A 21-year-old Caucasian man noticed enlargement of his left testis and underwent orchiectomy. The testis was largely replaced by a circumscribed predominantly solid $7 \mathrm{~cm}$ tumor. The cut surface ranged from gray to yellow-brown and contained scattered small cysts.

The low-power appearance was dominated by lobules of loose edematous stroma containing variable numbers of embryoid bodies (Figure 3), usually one to three but occasionally more (Figure 3c). Focally the lobules appeared to fuse forming more expansile aggregates but the constituent components of the embryoid body (germ disc and yolk-sac epithelium) could still be appreciated. The lobules were separated from one another by nonspecific paucicellular fibrous stroma; prominent foci of hemorrhage were present in areas. The majority of the embryoid bodies were wellformed with easily recognizable hyperchromatic embryonal carcinoma-type epithelium representing the germ disc associated with yolk-sac epithelium (Figure 3c). Superior to the germ disc was a wellformed cavity, reminiscent of the amniotic cavity within a developing embryo. The yolk-sac epithelium often imperceptibly merged with the loose edematous stroma of each lobule. Cysts reflective of the yolk-sac cavity were only occasionally evident but rarely underwent prominent dilation. The remaining 20\% of the tumor was composed of roughly equal amounts of yolk-sac tumor, embryonal carcinoma, and teratoma, including both mature and immature elements (Figure 4$)$. A very minor $(<1 \%)$ seminoma component was seen, but definitive germ cell neoplasia in situ was not identified, although significant 

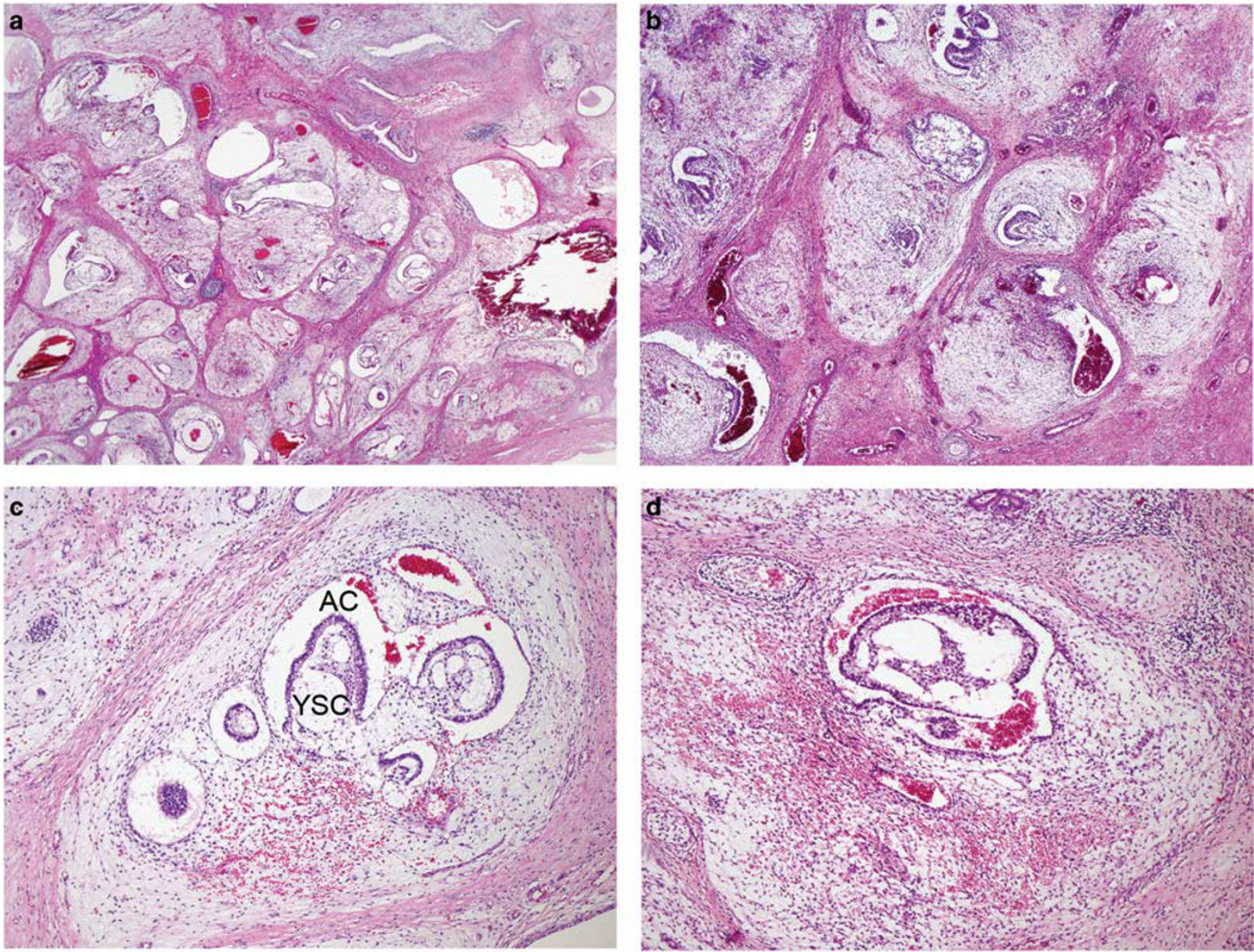

Figure 3 Representative low to medium power views of hematoxylin and eosin stained sections of a testicular polyembryoma (Case 2). The embryoid bodies are evenly distributed in a multinodular pattern with scattered variably sized cysts, which are sometimes filled with hemorrhage (a, b). The aggregates of embryoid bodies are embedded in a loose edematous to myxoid stroma, which together form lobules that are accentuated by the surrounding fibrous background (a-c). Several embryoid bodies, especially those seen on higher power (c,d), contain crescentic to cystic spaces which recapitulate the amniotic cavity and yolk-sac vesicle. The amniotic cavity (AC) and yolk-sac cavity (YSC) of one embryoid body are highlighted in $\mathbf{c}$.

uninvolved testis was not present for evaluation. The tumor was confined to the testis without evidence of lymphovascular invasion. It is unclear whether the patient received subsequent therapy, but he was alive and well 40 years later.

\section{Discussion}

Only a small number of gonadal tumors with a polyembryoma component have been reported in any detail ${ }^{5-22}$ since Peyron's seminal observations in the 1930s. ${ }^{1}$ Two cases we have seen (both ovarian) have been documented elsewhere, ${ }^{14-16}$ and along with the two testicular cases described here for the first time account for one quarter of the reported examples of this unique entity. Like others, we have been intrigued by the remarkable morphology of this neoplasm, prompting our recent review of the topic. ${ }^{1}$
It is the purpose of this communication to add to knowledge of this tumor by fully documenting two new cases, to review in detail information concerning the occurrence of this neoplasm in both gonads, and to discuss the opposing opinions on where this process belongs within the category of germ cell tumors. We also highlight what is surely one of the most remarkable neoplastic formations, the embryoid body whether seen in the gonads as in our cases (Figure 5a and b), at metastatic sites (Figure 5c), or as a significant component of a primary extragonadal germ cell tumor (Figure 5e). We also compare its morphology with that of an early embryo (Figure $5 \mathrm{~d}$ ), and comment briefly on its immunohistochemical staining characteristics (Figure $5 \mathrm{f}$ ).

As we are reporting two testicular tumors and the first case studied by Peyron was from the male gonad, we begin our literature review by considering testicular cases with comment on the two cases 

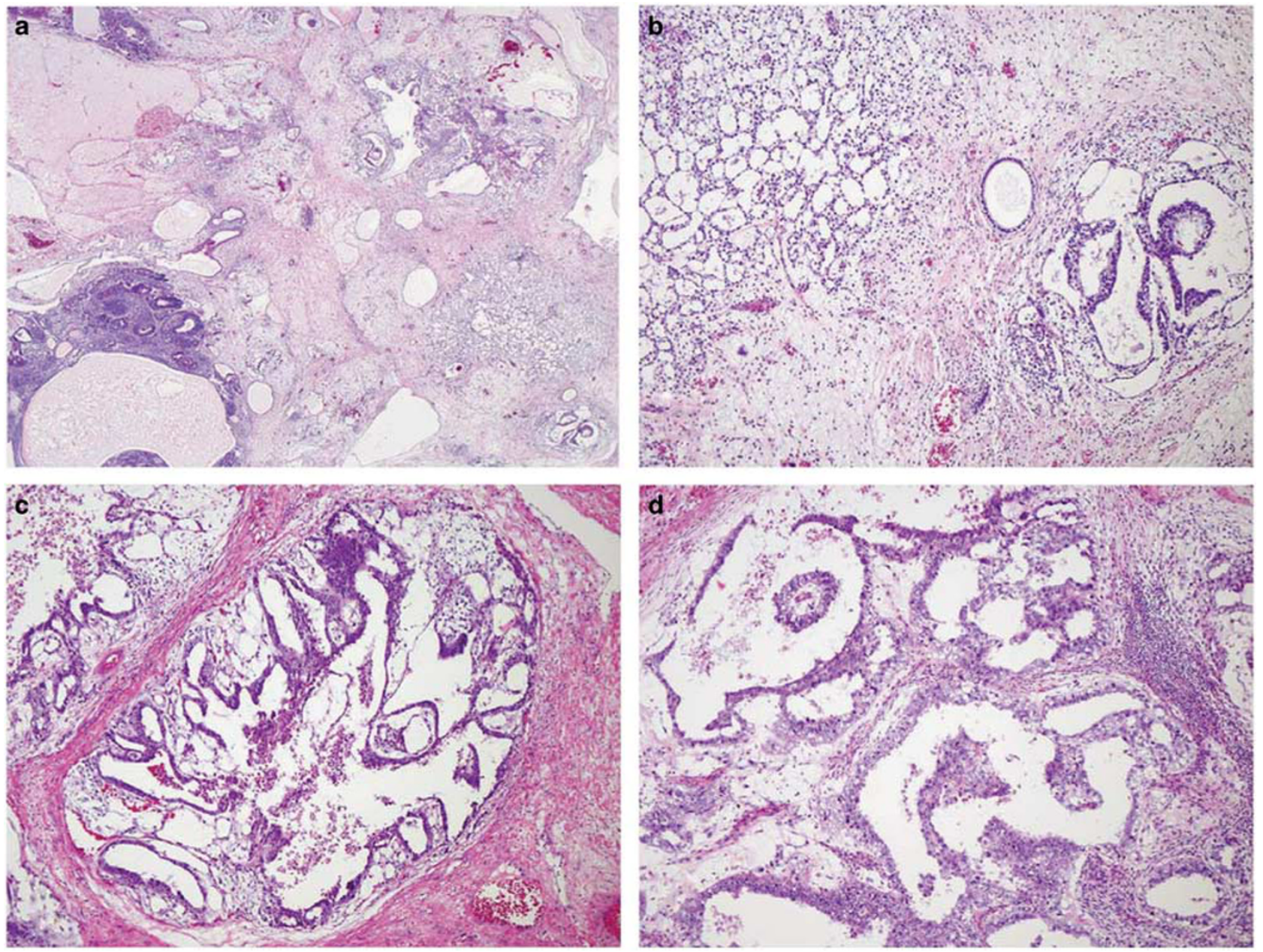

Figure 4 Representative hematoxylin and eosin stained sections of the varied germ cell components that may be seen in association with a polyembryoma (Case 2). Mature (not pictured) and immature teratomatous elements (a, bottom left) may be present. The yolk-sac epithelium, which comprises a portion of the embryoid body, may proliferate into larger aggregates, forming a yolk-sac tumor component (a, bottom right b, left). The embryoid bodies may fuse to form an expansile aggregate (c). Additional germ cell components, such as embryonal carcinoma (d), may also be present.

newly reported here. We then discuss ovarian examples before commenting on the appearances seen in tumors with a polyembryoma component. The presence of both yolk-sac and embryonal carcinoma-type epithelium within the embryoid body may bring forth a wide array of appearances due to the potential for them to proliferate to form varying patterns and amounts of yolk-sac tumor and embryonal carcinoma. These may be associated with embryoid bodies in various amounts and forms, and in some cases with other germ cell tumor components such that individual tumors may be remarkably complex.

\section{Testis}

Although the existence of embryoid bodies in some testicular tumors dates back to the work of Peyron over eight decades ago, broad interest in them and in neoplasms in which they dominate-polyembryoma -was slow to evolve. The classic paper of Friedman and Moore ${ }^{23}$ contains two illustrations of embryoid bodies said to be present within embryonal carcinoma. That paper must be one of the first, maybe even the first, to use the specific English language term-embryoid body. It is not clear to us who first used the term 'Polyembryoma.' It appears in the first World Health Organization (WHO) classification of testicular tumors published in $1977^{24}$ and was used from early in those deliberations, as it appears in material provided to us by the late Dr Robert E Scully representing the results of a meeting held in Zurich from December 13 to 15, 1971. The same year, Dr Gunnar Teilum used the term in the first edition of his book, ${ }^{25}$ and a year later the French investigator Gaillard, ${ }^{26}$ used it in a paper focusing on the patterns of yolk-sac tumor seen in tumors with a polyembryoma component. Appropriately, the latter paper appeared in a special issue of a Scandinavian journal in tribute to Teilum whose seminal contributions on the yolk-sac tumor, which on occasion is observed to 

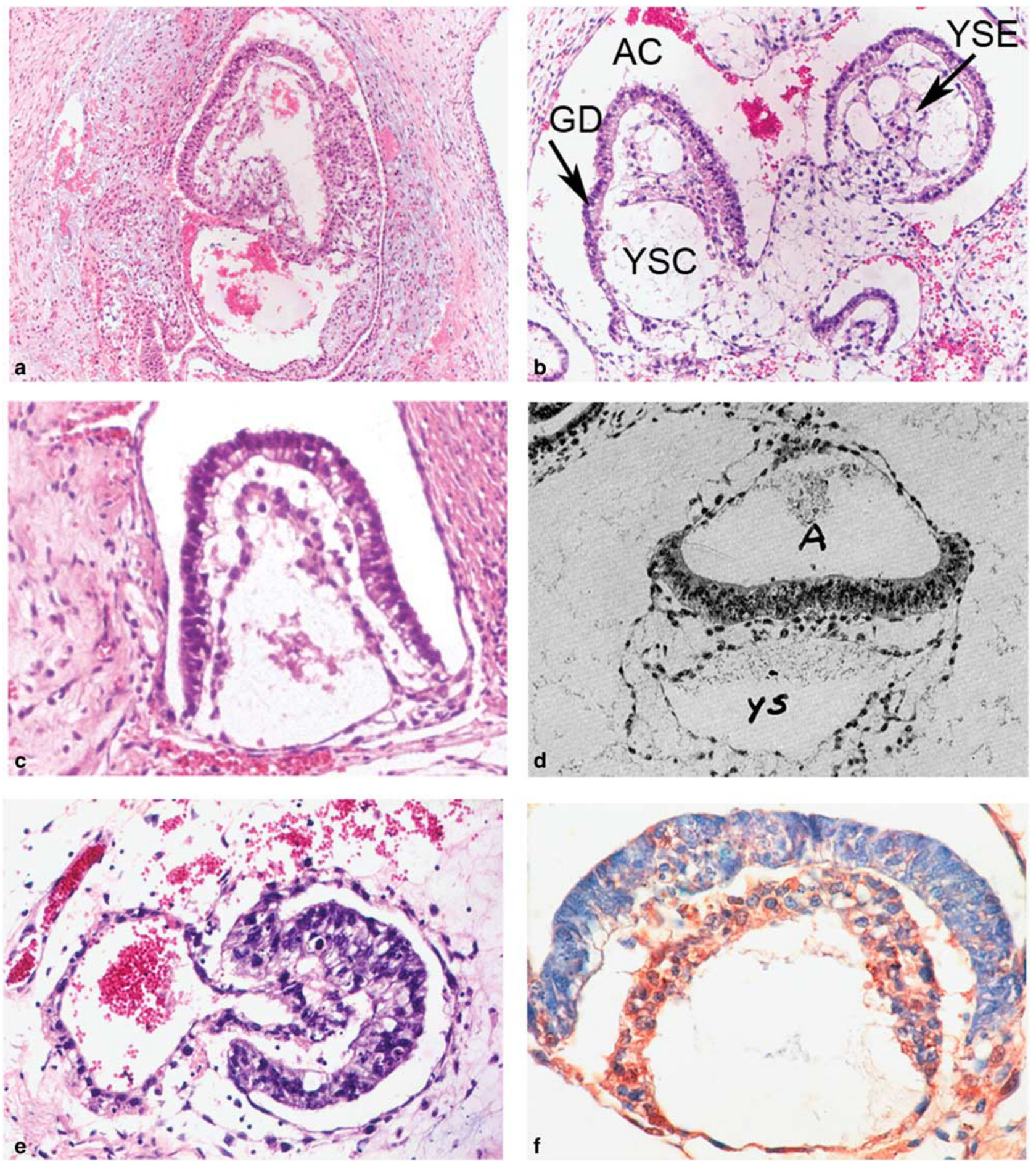

Figure 5 Representative depictions of embryoid bodies (a-c, e, f) and the early embryo whose structure they recapitulate (d). Well-formed embryoid bodies were present in both testicular cases reported here $(\mathbf{a}, \mathbf{b})$. An embryoid body may be a component of metastasis, as seen in a case of a testicular germ cell tumor metastatic to lymph nodes (c). In addition to gonadal locations, polyembryomas and the embryoid body that composes this neoplasm may be seen at extragonadal sites such as the posterior mediastinum (e). These illustrations all demonstrate the distinct components of the embryoid body, which are defined by a well-formed hyperchromatic germ disc (GD) with underlying less conspicuous delicate yolk-sac epithelium (YSE) and distinct embryologic cavities. The space superior to the germ disc recapitulates the amniotic cavity (AC), while the cavity beneath the yolk-sac epithelium recapitulates the yolk-sac cavity (YSC). By immunohistochemistry, the embryoid body demonstrates expression of alpha-fetoprotein (f) in the yolk-sac epithelium with only artifactual faint staining in the germ disc. A in d indicates amniotic cavity and YS, yolk sac cavity. 
arise directly from embryoid bodies, are well known and were summarized recently. ${ }^{27}$

One of the most definitive studies on embryoid bodies in testicular neoplasms was by Jacobsen who found that when extensively examined $42 \%$ of testicular mixed germ cell tumors contained embryoid bodies. ${ }^{2}$ She did not record their prominence in individual cases but our own large experience with testicular tumors indicates that although, as her figure indicates not rare, they are usually a minor component. In her book with Talerman, ${ }^{28}$ they comment that embryoid bodies are more frequent in testicular than in ovarian germ cell tumors. This may be true but we are not aware that anyone has searched as diligently for embryoid bodies in a large series of ovarian tumors as Jacobsen did in her study of testis cases. The frequency of the finding of embryoid bodies in cases of testicular embryonal carcinoma and yolk-sac tumor has varied. In a recent large series of embryonal carcinomas, they were found in $6 \%$ of the cases. ${ }^{29}$ In a series of yolk-sac tumors from infants and children, embryoid bodies were not found, ${ }^{30}$ possibly reflective of the differing nature of testicular yolk-sac tumors in children and adults.

It is difficult to comment with certainty on the prominence of embryoid bodies in some reported cases of 'polyembryoma' due to limited information on the extent of the embryoid body component. However, as best we can determine, in addition to the two cases we report with dominant polyembryoma components, the literature contains five other testicular examples. ${ }^{5-9}$ One of the papers just cited contains two potential cases but we only include Case 3 of that series as the other tumor appears to have had only a minor polyembryoma component. ${ }^{8}$

In the seven documented testicular cases, the patients ranged from 28 to 60 years of age with three being in their 20s, three in their early to mid-40s, and one a 60 year-old man with a history of Hodgkin's lymphoma. With the exception of one patient who presented with gynecomastia, ${ }^{5}$ the clinical presentation is that of a testicular mass. In the case with gynecomastia, the tumor had a component of choriocarcinoma, and the patient rapidly developed pulmonary metastases and died of disease three months following initial diagnosis. Follow-up of significant duration is available for four other patients: one was alive at 19 months, ${ }^{8}$ another at 5 years ${ }^{6}$ and one of our two patients was alive without disease at 40 years. Our other patient developed metastatic disease but long-term follow-up is not available.

The testicular tumors affect the left and right testis approximately equally, and the tumors are typically large $\left(6 \mathrm{~cm}\right.$ or more). Two tumors ${ }^{7,8}$ had a solid hemorrhagic cut surface, similar to our Case 1, which at the time of diagnosis was thought to potentially be a choriocarcinoma by an experienced urologist albeit it was not as diffusely hemorrhagic and friable as classic choriocarcinoma. As this appearance is virtually identical to one of the ovarian examples we have reviewed (illustrated in Ref. 1), these gross characteristics seem typical of a tumor that is in large part polyembryoma, although it is by no means always seen.

The morphologic features of the polyembryoma component in our two cases accords to that described in the literature but are briefly reviewed here. The unit that constitutes this neoplastic component, the embryoid body, is a generally rounded to oval formation with a central germ disc composed of embryonal carcinoma-type epithelium beneath which is a thin wispy layer of yolk-sac epithelium. Superior to the germ disc is a cavity which recapitulates the amniotic cavity and inferiorly there is sometimes a cavity which recapitulates the yolk-sac vesicle. In many cases, the yolk-sac cavity is not seen but rather a small (definitionally $<3 \mathrm{~mm}$, see later) focus of proliferating yolk-sac epithelium merging with adjacent stroma is evident. The embryoid bodies vary from perfectly to poorly formed, more being of the latter nature. The yolk-sac cavity in particular may result in conspicuous cyst formation. Striking hemorrhage is frequent, correlating with the often hemorrhagic gross appearance. A prominent low-power feature in some cases is lobulation due to the embryoid bodies being surrounded by a thick cuff of edematous to myxoid stroma (Figure 3a and b). This aspect has certainly been illustrated and described by others $^{13,15,17,28}$ but in the literature overall has perhaps not received the emphasis it merits. An already complicated histologic appearance is made even more so by the presence in all known cases of variable components of other germ cell tumor types as noted below. The two tumors reported herein are relatively similar with the exception of more prominent lobulated growth and more well-formed embryoid bodies in Case 2.

In addition to having a significant component of embryoid bodies, two tumors in the literature ${ }^{7,9}$ had a relatively minor teratomatous component as the only additional neoplastic element. One tumor only had an additional component of choriocarcinoma ${ }^{5}$ and in that regard differs from the remaining testicular neoplasms, all of which had two or more components-teratoma with embryonal carcinoma in two, $, 6,8$ teratoma with yolk-sac tumor in our Case 1 and teratoma with embryonal carcinoma and yolksac tumor in our Case 2. In summary, the associated other germ cell components were teratoma (five cases), embryonal carcinoma (three cases), yolk-sac tumor (one case) and choriocarcinoma (one case). Syncytiotrophoblast cells were present in two cases and hepatoid cells in one. For completeness, we note four additional cases in the literature in which mixed germ cell tumors contained minor amounts of embryoid bodies-Case 2 of Nakashima et al, ${ }^{8}$ the testicular case reported by Marin-Padilla ${ }^{10}$ and another report ${ }^{11}$ which is of interest because two brothers, each 17 years of age, had mixed germ cell tumors with a polyembryoma component. 


\section{Ovary}

Due to use of terminology not utilized today and limited descriptions, the literature on ovarian polyembryomas, like that of the testis, is not easy to evaluate. As with the testis, in the ovary we only consider tumors which appear to have had a dominant component of embryoid bodies but briefly note other reports where embryoid bodies were minor components in mixed germ cell tumors. In this analysis, we have excluded the case reported in 1945 from a two-year-old girl, ${ }^{12}$ which likely had a minor polyembryoma component. Soon thereafter the French-Canadian investigator Simard reported an ovarian tumor with embryoid bodies at sites of metastatic disease ${ }^{31}$ but a second paper by him, published in $1957,{ }^{13}$ contains the first well documented depiction of numerous embryoid bodies in a primary ovarian germ cell tumor. Overall, a total of nine definite ovarian cases are documented in the literature. ${ }^{13-22}$

The nine patients with ovarian polyembryoma have ranged from 9 to 43 years of age (mean 28). One presented with isosexual precocity, ${ }^{8}$ while the remainder presented with abdominal pain and/or pelvic mass. Significant follow-up information was available for five patients: one had metastatic disease at nine months, ${ }^{17}$ one was alive at one year, ${ }^{21}$ one at five years, ${ }^{15,16}$ one at nearly eight years ${ }^{8}$ and one at eleven years. ${ }^{17}$ Six tumors were left sided and three right sided, and they ranged from 9.5 to $34 \mathrm{~cm}$ in greatest dimension. They have typically been either solid or solid with a minor cystic component; the solid portion has occasionally been hemorrhagic as noted above.

Microscopic examination revealed a teratomatous component in eight of the nine cases. The exception is the report by Beck et $a l^{14}$ of a tumor diagnosed as 'solid malignant teratoma of the ovary'; the authors commented '(the tumor) does not have any elements showing more advanced tissue differentiation,' presumptively meaning lack of usual teratomatous elements. Although the microscopic description of this tumor suggests that it may have been entirely composed of embryoid bodies, the case is in the September 1968 California Tumor Tissue Registry slide set (accession \#15609/12) and Dr Robert Scully's files on that case contain the diagnoses of many of those who reviewed the case as part of the registry proceedings. Most commented on the presence of embryonal carcinoma, so we have concluded that the tumor, albeit dominantly composed of embryoid bodies did have other elements including embryonal carcinoma and (based on Dr Scully's notes) likely yolk-sac tumor. The eight slides of this case currently available for our review show only embryoid bodies. The other (nonteratomatous) components present in the nine ovarian cases were yolksac tumor (three cases), embryonal carcinoma and choriocarcinoma (two cases each). Finally, one tumor was recorded as having 'teratocarcinoma' the old term for embryonal carcinoma and teratoma, but in a table the authors of that report note there was no embryonal carcinoma so the nature of the overall components is uncertain. ${ }^{8}$ Three tumors had syncytiotrophoblast cells and one hepatoid cells. ${ }^{15}$

The case reported by Prat et al, ${ }^{15}$ which we have had the opportunity to review, has also been reported by others. ${ }^{16}$ An additional tumor has also been the subject of two separate reports in the literature. ${ }^{8,22}$ The case of Duhig ${ }^{32}$ is often cited when this topic is reviewed but we have not included it in our tabulation of the literature, as it is not convincing for having a dominant polyembryoma component. A similar comment, or lack of sufficient data, also pertains to three other cases-Case 7 of Kurman and Norris, ${ }^{33}$ Case 1 of Breen and Neubecker ${ }^{34}$ and the case of Tsukahara et al. ${ }^{35}$

\section{Comments on Polyembryoma in Both Gonads}

The experience we have just summarized enables the following comments to be made about the neoplasm under consideration. As it is a form of primitive germ cell neoplasia it, as expected, occurs mostly in the young in both sexes but has not been documented in a pre-pubertal boy. In females one has occurred in a 9 year-old. The other ovarian cases have occurred in the reproductive age group, and most of those in the testis have occurred in the typical age span of testicular germ cell tumors. The one outlier, a 60 year-old, is an uncommon but not unheard of age for a testicular germ cell tumor, which may be seen in later life. As with other germ cell tumors, they are usually non-functioning but there are rare exceptions, particularly when there is a component of choriocarcinoma or syncytiotrophoblast cells, which may result in hCG production. The tumors are generally large, more so in females in whom gonadal tumors are generally larger than in the male due to the later clinical presentation of an intra-abdominal as opposed to intra-scrotal mass. It is hard to comment on the natural history of the neoplasm because of the typical treatment instituted in recent decades, which is often effective in controlling malignant germ cell tumors. In addition, as there is no known pure tumor, the outcome based on the polyembryoma component alone cannot be commented upon other than the self evident statement that as it is primitive germ cell neoplasia, left untreated, the majority of patients would probably develop recurrence and in many cases die. That having been said 4 of the females were alive at intervals of 1 to 11 years, and 3 males at 19 months to 40 years, a better outcome than one might have predicted but the numbers are too small and information on treatment not detailed enough for definitive conclusions. We did not see lymphatic or blood vessel invasion in our cases and it has not been noted in the literature as best it can be evaluated so one could speculate this has an impact on a good prognosis in some cases. That having been said we have seen, and indeed previously documented, 
embryoid bodies in a blood vessel and in another case striking embryoid bodies in lymph nodes; ${ }^{1}$ in neither of those cases were embryoid bodies conspicuous enough in the primary tumor to warrant designation as polyembryoma. The gross appearance is often complicated by the presence of other elements but it does seem as if tumors with a prominent polyembryoma component are often solid red to brown and hemorrhagic.

In both sexes, the microscopic appearance of the tumor is complicated by the usual presence of a teratomatous component and in some cases by nonteratomatous elements, most often embryonal carcinoma or yolk-sac tumor but occasionally choriocarcinoma. The association with yolk-sac tumor is easily explained by yolk-sac epithelium being part of the embryoid body, and in one series 2 of 26 yolk-sac tumors were associated with embryoid bodies. ${ }^{36}$ The association with a teratomatous element is in line with the concept of some to consider the polyembryoma the most primitive form of teratomatous neoplasia. However, others consider it a distinctive form of mixed germ cell tumor. These contrasting opinions, enigmatically, were present in the companion recent WHO classifications of ovarian $^{4}$ and testicular germ cell tumors, ${ }^{3}$ respectively. That two authoritative groups could reach these differing conclusions speaks for itself considering the provocative issue posed by the remarkable association of yolk-sac epithelium and embryonal carcinoma in the embryoid body. An honest difference of opinion is probably no better justified than in this instance. In another work co-authored by one of us, out of deference to the opinion of the senior author, RHY accepted considering this a 'distinctive mixed germ cell tumor' ${ }^{37}$ As yolk-sac epithelium and embryonal carcinoma epithelium are intimately admixed in a tumor that, to date, has always had other germ cell elements, to consider the polyembryoma a pattern of mixed germ cell neoplasia is quite reasonable. Nonetheless, we believe the unique nature of this neoplasm should merit it being mentioned when conspicuous in individual neoplasms and looked at from another perspective it can be viewed as a primitive form of teratomatous neoplasia, the concept held by the late Dr Robert E. Scully. That embryoid bodies are so often present on the background of teratomatous elements is in accord with this approach as is the fact that the designation immature teratoma is applied to neoplasms having an embryonal character to their tissues (not just simply 'immature tissues of fetal type') and Dr Scully on several occasions commented to one of us (RHY) that as an embryoid body recapitulates a primitive structure of early embryonic life, tumors composed of significant numbers of them are logically considered primitive teratomatous neoplasia. We have rarely seen an isolated embryoid body, or small numbers of them, in teratomas without enough overall primitive tissue to be considered high-grade but most of the time when seen in a teratoma there are features that result in a high-grade immature teratoma diagnosis (assuming one accepts the concept of Dr Scully). As the embryonal carcinoma-type and yolk-sac-type epithelium are arranged in an organized way in a distinct unit he did not feel these components invasive embryonal carcinoma or yolksac tumor, respectively. Of course, as usually associated with other elements they are typically part of a mixed germ cell tumor. In his only peerreviewed contribution on polyembryoma he defined it as a 'germ cell tumor composed exclusively or almost exclusively of hundreds of embryoid bodies in varying stages of development'. ${ }^{15}$ He would make a second diagnosis of yolk-sac tumor or embryonal carcinoma when such elements proliferated in an expansile manner beyond the confines of the embryoid body to reach an arbitrary size of $3 \mathrm{~mm}$, an approach he used for diagnosing those elements in mixed germ cell tumors of the gonads in general. ${ }^{38}$

Embryoid bodies are classically rounded structures which when perfectly formed have a central darkly staining so-called germ disc composed of epithelium resembling that of embryonal carcinoma under which lies a thin layer of yolk-sac epithelium (Figure 5). These two paired epithelial elements are bounded superiorly and inferiorly by cavities recapitulating the amniotic cavity and yolk-sac vesicle. When numerous, embryoid bodies are often associated with a relatively prominent often myxoid stroma within which they often lie more or less centrally and a lobulated low-power appearance may be striking (Figure 3a and b). The overall appearance of embryoid bodies varies because deviations from the 'classic' perfectly formed structure are common and varying degrees of fragmentation of the embryoid body result in the germ disc epithelium and that of the yolk-sac vesicle being more haphazardly arranged. As Talerman noted, 'These atypical embryoid bodies may contain two or more amniotic cavities and share a single yolk-sac, or vice versa. The two cavities may vary in size and may be distorted'. ${ }^{39}$ Jacobsen and Talerman ${ }^{28}$ among others, have mentioned the occasional presence of individual mucinous glands representative of the allantois, which sometimes are misconstrued as teratomatous glands. A number of writers have commented on blastocyst-like bodies, as well as embryoid bodies being present in polyembryomas but we have not been struck with the former in the four cases we have had the opportunity to review. Neither have we been able to appreciate any difference in the morphology of the polyembryoma components in the two testis cases we have seen compared to the two ovarian cases; nor does the literature speak to any differences. Immunohistochemical studies of polyembryoma are limited but in a few cases staining of the yolk-sac epithelium for alphafetoprotein has, as expected, been shown. ${ }^{8,15,17,21,29,35}$ We illustrate this from the case of Prat et $a l^{15}$ (Figure 5f) and a companion stain for human chorionic gonadotrophin in this case (not illustrated) showed positive 
staining of the germ disc, but not of the yolk-sac epithelium.

The differential diagnosis of polyembryoma is limited. As the yolk-sac vesicle of some cases of polyembryoma may become cystically dilated it is worth brief comment that such a pattern should be distinguished from the rare polyvesicular vitteline pattern of yolk-sac neoplasia. ${ }^{40}$ The polyvesicular vitteline pattern is not associated with remnants of germ disc as is typical when cystic yolk-sac vesicles are seen in a polyembryoma, and the eccentric constriction often present in the units of the polyvesicular vitteline neoplasm is not a feature of the embryoid body. Of course that the polyvesicular vitteline pattern of yolk-sac tumor might co-exist with embryoid bodies is possible and has happened in one case. ${ }^{15}$ If only because of some overlap in nomenclature, it should be noted that the diffuse embryoma pattern of germ cell neoplasia differs from that of the polyembryoma inasmuch as the drapelike (necklace-like) arrangement of embryonal carcinoma and yolk-sac epithelium in the diffuse embryoma ${ }^{41}$ differs from the very distinctive organization of those elements in association with amniotic and yolk-sac cavities in the embryoid body of the polyembryoma. In addition the embryonal carcinoma and yolk-sac elements in the diffuse embryoma, although associated, grow together in an infiltrative manner and are not as crisply separated and arranged in such a distinct organized manner as embryoid bodies often are in a polyembryoma. We submit that the diffuse embryoma is a pattern of mixed germ cell neoplasia more definitively than is the polyembryoma.

It is uncertain whether a tumor composed only of embryoid bodies has occurred although the possibility surely exists. If one accepts the concept that embryoid bodies represent primitive teratomatous neoplasia the designation polyembryoma (or highgrade immature teratoma with prominent polyembryoma component) is appropriate when the only components in addition to the embryoid bodies are typical teratomatous ones. Such are rare but a few cases definitively or probably fall in this categorytwo testicular ${ }^{7,9}$ and two ovarian. ${ }^{18,20}$ The presence on their own, or additionally, of invasive nonteratomatous elements in a tumor with embryoid bodies would place the polyembryoma in such cases within a mixed germ cell tumor. ${ }^{3}$

In conclusion, the polyembryoma is a distinctive pattern of primitive germ cell neoplasia best considered, in the opinion of some, the most immature form of teratomatous neoplasia, but by others a distinctive pattern of mixed germ cell neoplasia. It typically occurs in the gonads, with approximately equal frequency in each sex, but like other germ cell tumors can rarely be seen at extragonadal sites. ${ }^{42}$ Its age distribution equates to that of other primitive germ cell tumors as does, most likely, the behavior. The tumor is fascinating because of the presence in neoplasia of a formation similar to that of an embryo circa 13-18 days of gestation (Figure 5 d). ${ }^{43}$ Identification of polyembryoma by the pathologist may have several practical points in addition to mere academic interest prompted by the remarkable morphology: (1) explanation of an elevated AFP level, (2) prompt further sampling to identify foci of yolk-sac tumor or embryonal carcinoma, (3) recognizing embryoid bodies in such cases may help explain unusual patterns of neoplasia as falling in the embryonal carcinoma or yolk-sac categories, and (4) awareness of the morphology will aid identification at extragonadal primary and metastatic sites.

\section{Acknowledgments}

Dr Lars Egevad kindly assisted in obtaining recuts of Case 2, on which Dr Robert Scully had consulted many years ago. Dr Jaime Prat took the photograph from which Figure 5c comes and kindly gave it to us. Figure $5 \mathrm{~d}$ is a slightly modified version of a 16-day embryo illustrated by Dr Arthur Hertig in one of his classic papers on early development of the embryo (Figure 17 of Ref. 43). Dr Stall was formerly the Surgical Pathology Fellow for the Robert E. Scully Collection and the Robert E. Scully Fellow in Gynecologic Pathology, both at the Massachusetts General Hospital, Harvard Medical School.

\section{Disclosure/conflict of interest}

The authors declare no conflict of interest.

\section{References}

1 Young RH, Stall JN, Sevestre H. The polyembryoma. One of the most intriguing human neoplasms, with comments on the investigator who brought it to light, Albert Peyron. Int J Gynecol Pathol 2016;35:93-105.

2 Jacobsen GK. Histogenetic considerations concerning germ cell tumours. Morphological and immunohistochemical comparative investigation of the human embryo and testicular germ cell tumours. Virchows Arch 1986;408:509-525.

3 Moch H, Humphrey PA, Ulbright TM, et al. WHO Classification of Tumours of the Urinary System and Male Genital Organs. IIARC Press: Lyon, 2016, pp. 216.

4 Kurman RJ, Carcangiu ML, Herrington CS, et al. WHO Classification of Tumours of Female Reproductive Organs. IARC Press: Lyon, 2014, pp. 61.

5 Melicow MM. Embryoma of testis. Report of case and a classification of neoplasms of the testis. J Urol 1940;44: 333-344.

6 Evans RW. Developmental stages of embryo-like bodies in teratoma testis. J Clin Path 1957;10:31-39.

7 Cornet A, Bultinck J, Declercq G. Polyembryoma of the testis. Acta Urol Belg 1985;53:107-114.

8 Nakashima N, Murakami S, Fukatsu T, et al. Characteristics of 'embryoid body' in human gonadal germ cell tumors. Hum Pathol 1988;19:1144-1154. 
9 Carlomagno N, Nastro P, Lombari P, et al. Polyembryoma of the testis: case report following chemotherapy for non-Hodgkin’s lymphoma. G Chir 2002;23:65-70.

10 Marin-Padilla M. Origin, nature and significance of the 'embryoids' of human teratomas. Virchows Arch Path Anat 1965;340:105-121.

11 Bakaris S, Resim S, Tunali N. Testicular mixed germ cell tumor with polyembryoma component in brothers. Pediatr Dev Pathol 2005;8:92-97.

12 Nicod JL. Phénomènes de parthénogénèse dans des tumeurs de l'homme. Bull Soc Vaud Sci Nat 1945;62: 495-505.

13 Simard LC. Polyembryonic embryoma of the ovary of parthenogenetic origin. Cancer 1957;10:215-223.

14 Swanson Beck J, Fulmer HF, Lee ST. Solid malignant ovarian teratoma with 'embryoid bodies' and trophoblastic differentiation. J Path 1969;99:67-73.

15 Prat J, Matias-Guiu X, Scully RE. Hepatic yolk sac differentiation in an ovarian polyembryoma. Surg Pathol 1989;2:147-150.

16 Chapman DC, Grover R, Schwartz PE. Conservative management of an ovarian polyembryoma. Obstet Gynecol 1994;83:879-882.

17 King ME, Hubbell MJ, Talerman A. Mixed germ cell tumor of the ovary with a prominent polyembryoma component. Int J Gynecol Pathol 1991;10:88-95.

18 Yao G, Gao H, Hao Y. Histopathological and immunohistochemical study of ovarian polyembryoma. Zhonghua Fu Chan Ke Za Zhi 1995;30:18-21.

19 Nishida T, Oda T, Sugiyama T, et al. Ovarian mixed germ cell tumor comprising polyembryoma and choriocarcinoma. Eur J Obstet Gynecol 1998;78:95-97.

20 Takemori M, Nishimura R, Yamasaki M, et al. Ovarian mixed germ cell tumor comprised of polyembryoma and immature teratoma. Gynecol Oncol 1998;69:260-263.

21 Jondle DM, Shahin MS, Sorosky J, et al. Ovarian mixed germ cell tumor with predominance of polyembryoma: a case report with literature review. Int J Gynecol Pathol 2001;21:78-81.

22 Takeda A, Ishizuka T, Goto T, et al. Polyembryoma of ovary producing alpha-fetoprotein and HCG: immunoperoxidase and electron microscopic study. Cancer 1982;49:1878-1889.

23 Friedman NB, Moore RA. Tumors of the testis-a report on 922 cases. The Military Surgeon 1946;99:573-593.

24 Mostofi FK. Histologic typing of testis tumours. International Histologic Classification of Tumours, no. 16. World Health Organization: Geneva, 1977.

25 Teilum G. Polyembryoma. In: Special Tumors of Ovary and Testis and Extragonadal Lesions. Comparative Pathology and Histological Identification. Munksgaard: Copenhagen, 1971, pp 158-159.

26 Gaillard JA. Yolk-Sac tumour patterns and entoblastic structures in polyembryomas. Acta Path Microbiol Scand 1972;80(Suppl. 233):18-25.

27 Young RH. The Yolk Sac Tumor: reflections on a remarkable neoplasm and two of the many intrigued by
it-Gunnar Teilum and Aleksander Talerman-and the bond it formed between them. Int J Surg Pathol 2014;22:677-687.

28 Jacobsen GK, Talerman A. Atlas of Germ Cell Tumours. Munksgaard: Copenhagen, 1989.

29 Kao CS, Ulbright TM, Young RH, et al. Testicular embryonal carcinoma. A morphologic study of 180 cases highlighting unusual and unemphasized aspects. Am J Surg Pathol 2014;38:689-697.

30 Cornejo KM, Frazier L, Lee RS, et al. Yolk sac tumor of the testis in infants and children: a clinicopathologic analysis of 33 cases. Am J Surg Pathol 2015;39: 1121-1131.

31 Simard LC. Tératôme malin total de l'ovaire humain d'origine parthénogénétique vraisemblable. Rev Canad de Biol 1950;9:344-365.

32 Duhig JT. An unusual adenocarcinoma of the ovary: a case simulating Schiller's 'mesonephroma'. Am J Obstet Gynecol 1959;77:201-205.

33 Kurman RJ, Norris HJ. Malignant mixed germ cell tumors of the ovary: a clinical and pathologic analysis of 30 cases. Obstet Gynecol 1976;48:579-589.

34 Breen JL, Neubecker RD. Malignant teratoma of the ovary. An analysis of 17 cases. Obstet Gynecol 1963;21: 668-681.

35 Tsukahara Y, Fukuta T, Yamada T, et al. Retroperitoneal giant tumor formed by migrating polyembryoma with numerous embryoid bodies from an ovarian mixed germ cell tumor. Gynecol Obstet Invest 1991;31:58-60.

36 Kawai M, Kano T, Furuhashi Y, et al. Prognostic factors in yolk sac tumors of the ovary: a clinicopathologic analysis of 29 cases. Cancer 1991;67: 184-192.37.

37 Ulbright TM, Young RH. Tumors of the testis and adjacent structures. In: Atlas of Tumor Pathology, Fourth Series, Fascicle 18, American Registry of Pathology: Silver Springs, Maryland, 2015, pp 214.

38 Young RH, Scully RE. Testicular Tumors. ASCP: Chicago, 1990, pp 45.

39 Talerman A. Polyembryoma in germ cell tumors. In: Pathology of the Testis and Its Adnexa. Contemporary Issues in Surgical Pathology, Chap. 3, Vol. 7. Churchill Livingstone: New York, 1986, pp 55-56.

40 Young RH, Ulbright TM, Policarpio-Nicolas MLC. Yolk Sac Tumor with a prominent polyvesicular vitelline pattern: a report of three cases. Am J Surg Pathol 2013; 37:393-398.

41 Cardoso de Almeida PC, Scully RE. Diffuse Embryoma of the testis: a distinctive form of mixed germ cell tumor. Am J Surg Pathol 1983;7:633-642.

42 Danon M, Weintraub BD, Kim SH, et al. Sexual precocity in a male due to thoracic polyembryoma. J Pediatr 1978;92:51-54.

43 Hertig A. On the development of the amnion and exocoelomic membrane in the pre-villous human ovum. Yale J Biol Med 1945;18:107-115. 